\title{
Idiopathic pulmonary fibrosis: securing a confident diagnosis for every patient
}

\author{
Mark G. Jones ${ }^{1,2}$, Simon L.F. Walsh ${ }^{3}$, Kirk D. Jones ${ }^{4}$ and Luca Richeldi ${ }^{1,2}$ \\ Affiliations: ${ }^{1}$ Academic Unit of Clinical and Experimental Sciences, University of Southampton Faculty of \\ Medicine, University Hospital Southampton, Southampton, UK. ${ }^{2}$ Southampton NIHR Respiratory Biomedical \\ Research Unit, University Hospital Southampton, Southampton, UK. ${ }^{3}$ Dept of Radiology, Kings College \\ Hospital Foundation Trust, London, UK. ${ }^{4}$ Dept of Pathology, University of California San Francisco, \\ San Francisco, CA, USA.
}

Correspondence: Luca Richeldi, University Hospital Southampton, Tremona Road, Southampton, S016 6YD, UK. E-mail: L.Richeldidsoton.ac.uk

0

@ERSpublications

Inconsistent UIP pattern on HRCT in patients with suspected IPF should not rule out possibility of an IPF diagnosis http://ow.ly/YcVao

Almost half a century ago Liebow and Carrington [1] proposed their landmark framework to classify the idiopathic interstitial pneumonias (IIPs), a diverse group of diffuse parenchymal lung diseases of unknown aetiology. This classification approach has since informed the principal that in IIP histopathological pattern may have clinical implications including prognosis and treatment. Five subgroups were identified, the most frequent of which was termed "usual" interstitial pneumonia (UIP) and is the pattern which, in the absence of any identifiable cause, is diagnostic of the prototypic chronic progressive fibrotic lung disease idiopathic pulmonary fibrosis (IPF) [2].

The current evidence-based guidelines for the diagnosis and management of IPF represent the combined effort of the American Thoracic Society, European Respiratory Society, Japanese Respiratory Society and the Latin American Thoracic Association and were published in 2011 [2]. This statement recommends multidisciplinary evaluation of patients with suspected IPF as the diagnostic gold standard. This approach involves an integration of clinical, radiological and, when lung biopsy material is available, pathological data. First, chest high-resolution computed tomography (HRCT) appearances are stratified based on three categories: UIP, possible UIP and inconsistent with UIP. In the correct clinical context a typical pattern of UIP on HRCT may be sufficient to diagnose IPF without the need for surgical lung biopsy. Therefore, HRCT plays a central role in the diagnosis of patients with suspected IPF and, once performed, greatly influences subsequent management decisions. If HRCT appearances are those of possible or inconsistent with UIP, surgical lung biopsy is required to secure the diagnosis of IPF. Biopsy appearances are classified into one of the four histopathological patterns defined as: UIP, probable UIP, possible UIP and not UIP. The final multidisciplinary diagnosis is then classified as IPF, probable IPF, possible IPF and not IPF.

Since the publication of the 2011 guidelines this diagnostic approach has faced several challenges, many of which relate to the stratification of patients into categories based upon diagnostic probabilities, a feature which sharply contrasts with diagnostic approaches to other lung diseases, such as asthma or lung cancer. In patients with classic appearances of UIP on HRCT, a rapidly progressive disease course and no identifiable triggers, making a confident diagnosis of IPF is straightforward without the need for multidisciplinary discussion. The difficulty arises when the presenting HRCT appearances are those of possible UIP or inconsistent with UIP, making the diagnosis of IPF less certain. Following the approval of the novel anti-fibrotic therapies nintedanib and pirfenidone for the treatment of IPF [3, 4], achieving an early, confident diagnosis of IPF has become particularly important. These treatments can only be prescribed for patients with a diagnosis of IPF, and delay in commencing treatment has the potential to reduce cumulative

Received: Feb 042016 | Accepted: Feb 042016

Conflict of interest: Disclosures can be found alongside the online version of this article at erj.ersjournals.com

Copyright @ERS 2016 
patient benefit. The availability of these therapies has, therefore, highlighted several challenges when applying current diagnostic guidelines to individual patients [3, 4]. As an example, a common diagnostic problem encountered is the IPF patient whose HRCT lacks honeycombing but otherwise has basal subpleural reticular abnormalities typical of UIP. Guideline recommendations mandate that surgical lung biopsy is needed to secure the diagnosis of IPF, yet a significant proportion of these patients are unable to undergo lung biopsy because of preclusive comorbidities. Ultimately, these patients may be denied access to anti-fibrotic treatments only licensed for use in patients whose diagnosis of IPF is certain.

Given these intrinsic limitations in the current guidelines, it would be desirable if HRCT criteria for a UIP pattern could be expanded to include those patients whose imaging appearances are not those of typical UIP but are otherwise consistent with UIP. This has been the focus of several studies over the past 3 years which broadly point to the same message that, in the correct clinical context, a coarse basal predominant reticular abnormality on HRCT without honeycombing is likely to be UIP in most patients. Reflecting this growing perception, HRCT inclusion criteria for a recent IPF drug trial permitted enrolment of patients with HRCT appearances that partly met possible UIP criteria [3]. There is a need for this to be considered for inclusion in future diagnostic recommendations; despite treatment guidelines for IPF being updated in 2015 to reflect therapeutic advances [5], there has not yet been an update on the diagnostic guidelines.

In this issue of the European Respiratory Journal, YAGIHASH et al. [6] report retrospective analyses on discordance between imaging and histological diagnoses of UIP from patient cohorts with a diagnosis of IPF enrolled in the multicentre clinical trials conducted by the Idiopathic Pulmonary Fibrosis Clinical Research Network in the USA. They evaluated rates of radiological-histological concordance and discordance in 241 patients. Whilst UIP on HRCT was, unsurprisingly, highly correlated with histological UIP, about one-third (31\%) of patients within this study population had HRCT findings inconsistent with UIP. Of these patients, almost all (95\%) had definite or probable UIP on histological evaluation [6].

However, these results must be interpreted with caution if one is to avoid two inaccurate conclusions. First, that $95 \%$ of patients with a HRCT read as inconsistent with UIP have UIP and secondly, that $94 \%$ of patients with possible UIP on HRCT actually have UIP. The astute reader will realise that this study population is not generalisable to patients being investigated for suspected IPF in clinical practice. This is a highly selected population of patients with an established diagnosis of IPF, taken from several clinical trial centres and without knowledge of the frequency of patients with inconsistent with UIP on HRCT who subsequently had a histological pattern other than UIP on biopsy; as a result, translating these findings directly to clinical practice is not possible.

However, this study does highlight an important issue. The finding that almost one-third of patients within this IPF population had a HRCT pattern inconsistent with UIP emphasises that IPF must still be considered to be within the differential diagnosis of such patients. Acknowledging this finding, the authors propose that the term "inconsistent with UIP" be removed from future guidelines [6]. One possible replacement would be the term "radiologically inconsistent with UIP", thus emphasising that in the absence of histopathological sampling UIP should not be confidently excluded.

Within this patient population interobserver agreement between radiologists was good (weighted kappa $=0.77$ ), yet still approximately one in 20 patients considered to have a UIP pattern on HRCT by one expert thoracic radiologist were considered to have an inconsistent with UIP pattern by a second expert radiologist. Recently, an international study evaluating observer agreement for the HRCT diagnostic categories defined in the 2011 guidelines reported only moderate agreement between 112 thoracic radiologists [7]. Surprisingly, this was not improved in radiologists with $>25$ years' experience. Whilst HRCT has a central role in diagnosis, similar patterns on HRCT may have different histological diagnoses and, as we enter an era of targeted anti-fibrotic therapy, it is unlikely that radiological pattern alone will be sufficient to diagnose and guide treatment.

In cases where HRCT is not diagnostic of UIP a surgical lung biopsy is advised. Current surgical practice using video-assisted thoracoscopic surgery has a mortality of $\sim 2-5 \%$ [8-10]. However, many patients being evaluated for IPF have significant physiological impairment or comorbidities and with concerns regarding surgical complications, a biopsy may not be considered safe. It is possible that less invasive diagnostic approaches, such as transbronchial lung cryobiopsy using a flexible bronchoscope, may underpin the development of future histopathological or molecular stratification approaches [11]. Another possible approach was recently demonstrated by KIM et al. [12] who proposed the development of a diagnostic genomic signature of UIP. A number of molecular markers of disease behaviour with possible diagnostic utility have recently been proposed. For example, histological analyses of $\alpha v \beta 6$-integrin expression, an epithelial restricted molecule implicated in multiple models of lung fibrosis, identified increased risk of death in patients with pulmonary fibrosis with the highest expression [13], and serum levels of the collagen cross-linkning enzyme lysyl oxidase-like 2 correlated with disease progression in patients with IPF [14]. 
Whilst these novel findings support the concept that molecular biomarkers may be diagnostically useful in IPF, a number of hurdles must be overcome if translation to routine clinical practice is to be achieved. One important consideration in these efforts is that patient selection variability, for example through centre to centre variability or tertiary versus secondary care referral, has the potential to significantly bias study findings, interpretation and general applicability. Ultimately, prospective multicentre longitudinal studies of all incident cases presenting with suspected IPF are required to better investigate clinical, radiological and molecular IPF phenotypes. This will provide the opportunity to increase our understanding of the natural history of chronic progressive fibrotic ILDs and to identify the factors that truly discriminate between distinct subtypes or endotypes of fibrotic lung disease with prognostic and treatment implications. Finally, in an effort to personalise treatments for IPF [15], progressing towards a more reliable and "consistent" diagnostic framework should ultimately help to identify additional safe and effective treatments for these patients.

\section{References}

1 Liebow AA, Carrington DB. The interstitial pneumonias. In: Simmon M, Potchen EJ, Le May M, eds. Frontiers of Pulmonary Radiology. New York, Grune \& Stratteon, 1969, pp. 102-141.

2 Raghu G, Collard HR, Egan JJ, et al. An Official ATS/ERS/JRS/ALAT Statement: Idiopathic Pulmonary Fibrosis: Evidence-based Guidelines for Diagnosis and Management. Am J Respir Crit Care Med 2011; 183: 788-824.

3 Richeldi L, Bois du RM, Raghu G, et al. Efficacy and safety of nintedanib in idiopathic pulmonary fibrosis. $N$ Engl J Med 2014; 370: 2071-2082.

4 King TE Jr, Bradford WZ, Castro-Bernardini S, et al. A phase 3 trial of pirfenidone in patients with idiopathic pulmonary fibrosis. N Engl J Med 2014; 370: 2083-2092.

5 Raghu G, Rochwerg B, Zhang Y, et al. An Official ATS/ERS/JRS/ALAT Clinical Practice Guideline: Treatment of Idiopathic Pulmonary Fibrosis. An Update of the 2011 Clinical Practice Guideline. Am J Respir Crit Care Med 2015; 192: e3-e19.

6 Yagihashi K, Huckleberry J, Colby TV, et al. Radiologic-pathologic discordance in biopsy-proven usual interstitial pneumonia. Eur Respir J 2016; 47: 1189-1197.

7 Walsh SL, Calandriello L, Sverzellati N, et al. Interobserver agreement for the ATS/ERS/JRS/ALAT criteria for a UIP pattern on CT. Thorax 2016; 71: 45-51.

8 Sigurdsson MI, Isaksson HJ, Gudmundsson G, et al. Diagnostic surgical lung biopsies for suspected interstitial lung diseases: a retrospective study. Ann Thorac Surg 2009; 88: 227-232.

9 Park JH, Kim DK, Kim DS, et al. Mortality and risk factors for surgical lung biopsy in patients with idiopathic interstitial pneumonia. Eur J Cardiothorac Surg 2007; 31: 1115-1119.

10 Hutchinson JP, Fogarty AW, McKeever TM, et al. In-hospital mortality following surgical lung biopsy for interstitial lung disease in the USA: 2000-2011. Am J Respir Crit Care Med 2015; [in press; DOI: 10.1164/ rccm.201508-1632OC].

11 Tomassetti S, Wells AU, Costabel U, et al. Bronchoscopic lung cryobiopsy increases diagnostic confidence in the multidisciplinary diagnosis of idiopathic pulmonary fibrosis. Am J Respir Crit Care Med 2015; [in press; DOI: 10.1164/rccm.201504-0711OC].

12 Kim SY, Diggans J, Pankratz D, et al. Classification of usual interstitial pneumonia in patients with interstitial lung disease: assessment of a machine learning approach using high-dimensional transcriptional data. Lancet Respir Med 2015; 3: 473-482.

13 Saini G, Porte J, Weinreb PH, et al. $\alpha \mathrm{v} \beta 6$ integrin may be a potential prognostic biomarker in interstitial lung disease. Eur Respir J 2015; 46: 486-494.

14 Chien JW, Richards TJ, Gibson KF, et al. Serum lysyl oxidase-like 2 levels and idiopathic pulmonary fibrosis disease progression. Eur Respir J 2014; 43: 1430-1438.

15 Britten N, Pope C, Halford S, et al. What if we made stratified medicine work for patients? Lancet Respir Med 2016; 4: 8-10. 